Prepared for the U.S. Department of Energy

under Contract DE-AC05-76RL01830

\title{
EMTA's Evaluation of the Elastic Properties for Fiber Polymer Composites Potentially Used in Hydropower Systems
}

\author{
Ba Nghiep Nguyen ${ }^{(1)}$ and Joshua Paquette ${ }^{(2)}$
}

(1) Pacific Northwest National Laboratory, PO Box 999, Richland, WA 99352

(2) Sandia National Laboratories, PO Box 5800, Albuquerque, NM 87185

August 2010

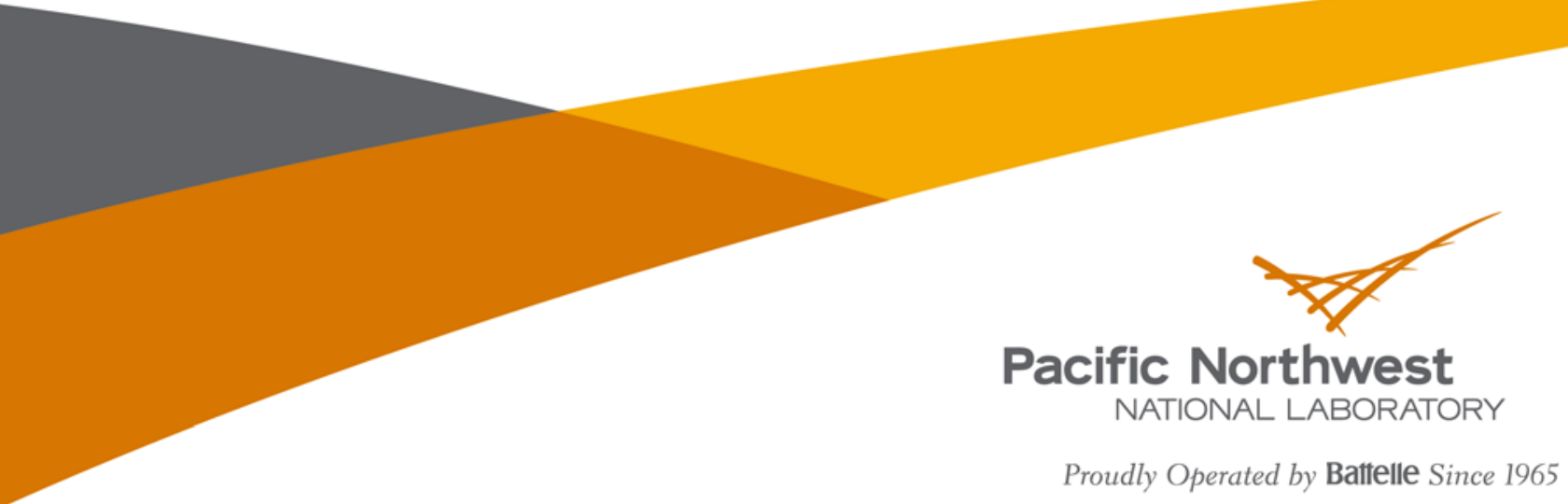




\section{DISCIAAIMER}

This report was prepared as an account of work sponsored by an agency of the United States Government. Neither the United States Government nor any agency thereof, nor Battelle Memorial Institute, nor any of their employees, makes any warranty, express or implied, or assumes any legal liability or responsibility for the accuracy, completeness, or usefulness of any information, apparatus, product, or process disclosed, or represents that its use would not infringe privately owned rights. Reference herein to any specific commercial product, process, or service by trade name, trademark, manufacturer, or otherwise does not necessarily constitute or imply its endorsement, recommendation, or favoring by the United States Government or any agency thereof, or Battelle Memorial Institute. The views and opinions of authors expressed herein do not necessarily state or rellect those of the United States Government or any agency thereof.

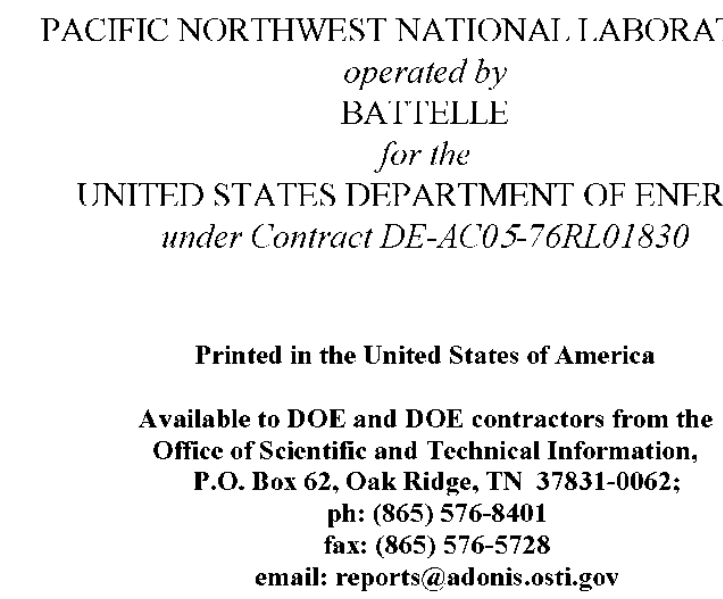

Available to the public from the National Technical Information Service, U.S. Department of Commerce, 5285 Port Royal Rd., Springfield, VA 22161 ph: (800) 553-6847 fax: (703) 605-6900 email: orders@ntis.fedworld.gov online ordering: http://www.ntis.gov/ordering.htm

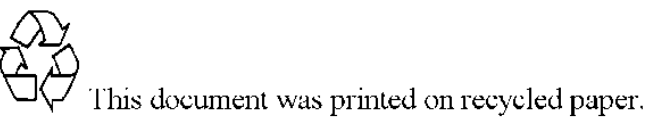

(9/2003) 
PNNL-19729

\title{
EMTA's Evaluation of the Elastic Properties for Fiber Polymer Composites Potentially Used in Hydr op ower Systems
}

\author{
Ba Nghiep Nguyen ${ }^{(1)}$ and Joshua Paquette ${ }^{(2)}$
}

(1) Pacific Northwest National Laboratory, PO Box 999, Richland, WA 99352

(2) Sandia National Laboratories, PO Box 5800, Albuquerque, NM 87185

August 2010

Prepared for

the U.S. Department of Energy

under Contract DE-AC05-76RL01830 


\section{Executive Summary}

Fiber-reinforced polymer composites can offer important advantages over metals where lightweight, cost-effective manufacturing and high mechanical performance can be achieved. To date, these materials have not been used in hydropower systems. In view of the possibility to tailor their mechanical properties to specific applications, they now have become a subject of research for potential use in hydropower systems. The first step in any structural design that uses composite materials consists of evaluating the basic composite mechanical properties as a function of the as-formed composite microstructure. These basic properties are the elastic stiffness, stress-strain response, and strength. This report describes the evaluation of the elastic stiffness for a series of common discontinuous fiber polymer composites processed by injection molding or by compression molding in order to preliminarily estimate whether these composites could be used in hydropower systems for load-carrying components such as turbine blades. To this end, the EMTA (Copyright (C) Battelle 2010) predictive modeling tool developed at the Pacific Northwest National Laboratory (PNNL) has been applied to predict the elastic properties of these composites as a function of three key microstructural parameters: fiber volume fraction, fiber orientation distribution, and fiber length distribution. These parameters strongly control the composite mechanical performance and can be tailored to achieve property enhancement. EMTA uses the standard and enhanced Mori-Tanaka type models combined with the Eshelby equivalent inclusion method to predict the thermoelastic properties of the composite based on its microstructure. 


\section{Contents}

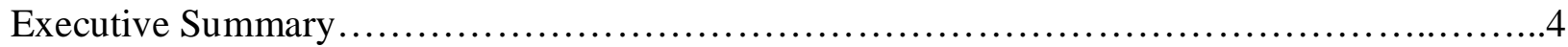

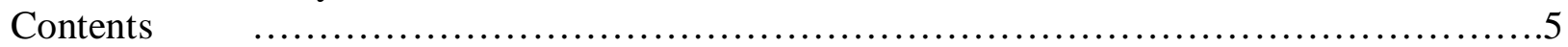

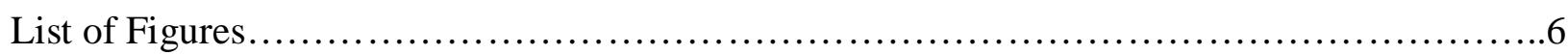

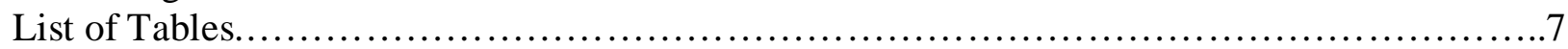

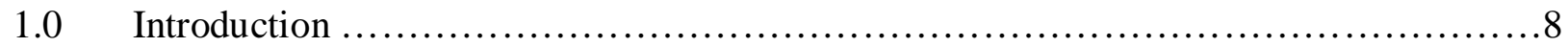

2.0 Processing Methods for Discontinuous Fiber Polymer Composites......................9

2.1 Injection Molding of Fiber-Filled Thermoplastics.................................9

2.2 Compression Molding of Fiber Preforms ....................................... 10

3.0 The Eshelby-Mori-Tanaka Approach and EMTA Software............................11

3.1 The Standard Eshelby-Mori-Tanaka Model......................................12

3.2 An Eshelby-Mori-Tanaka Model for Concentrated Composites.........................13

3.3 Solution for Misoriented Fiber Composites.....................................13

3.4 Solution for Composites with Fiber Length Distribution............................14

$4.0 \quad$ Numerical Applications..................................................... 15

4.1 EMTA Computation of Elastic Properties for Injection-Molded Fiber Thermoplastics....15

4.1.1 Results for Glass/PP .......................................................... 17

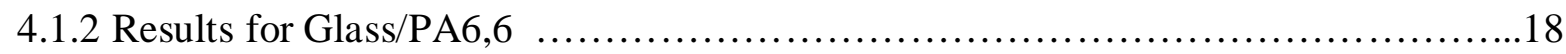

4.1.3 Results for Glass/PEEK ................................................18

4.1.4 Results for Carbon/PP, Carbon/PA6,6, and Carbon/PEEK $\quad \ldots \ldots \ldots \ldots \ldots \ldots \ldots \ldots \ldots . . .22$

4.2 EMT A Computation of Elastic Properties for Compression-Molded Fiber Thermosets...24

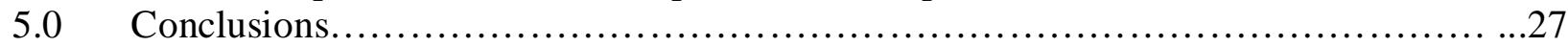

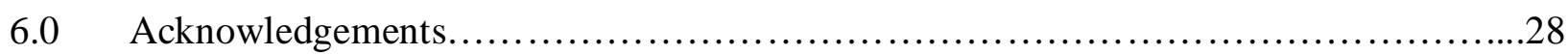

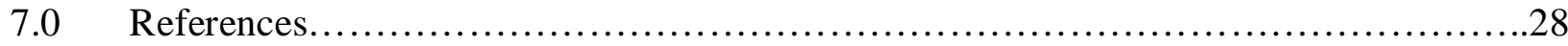




\section{List of Figures}

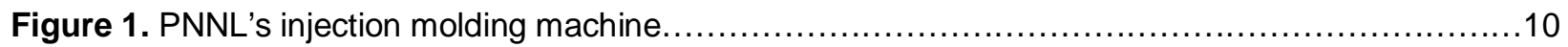

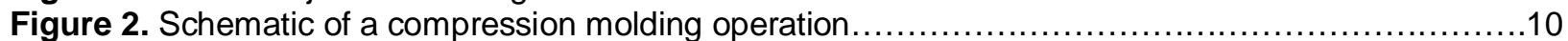

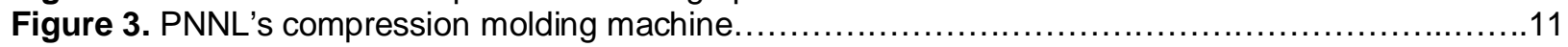

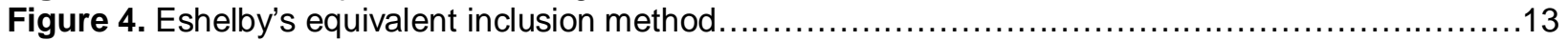

Figure 5. Theoretical definitions of the second- and fourth-order orientation tensors in terms of the

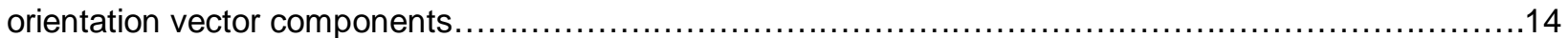
Figure 6. (a) A micrograph of a cross section of an injection-molded fiber material showing the orientation layer structure, and (b) Typical through-thickness variation of the $A_{11}$ component in a long-fiber-

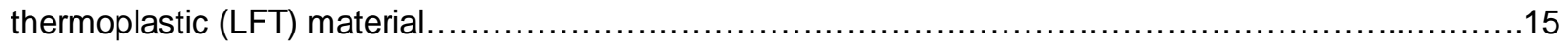

Figure 7. Assumed fiber orientation distribution for the EMTA calculation of the elastic properties for

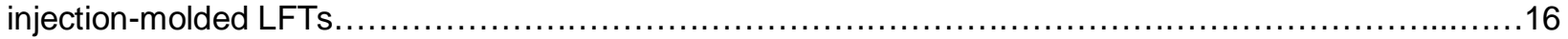
Figure 8. Assumed fiber length distributions for the EMTA calculation of the elastic properties for

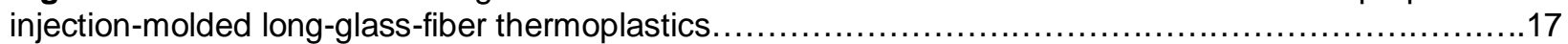
Figure 9. Predicted elastic properties for the glass/PP composites as a function of fiber volume fraction $v$ and fiber length distribution represented by the weight-average length: (a) Moduli $E_{11}, E_{22}$, and $E_{33}$, (b)

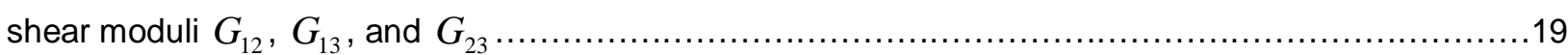
Figure 10. Predicted elastic properties for the glass/PA6,6 composites as a function of fiber volume fraction $v$ and fiber length distribution represented by the weight-average length: (a) Moduli $E_{11}, E_{22}$,

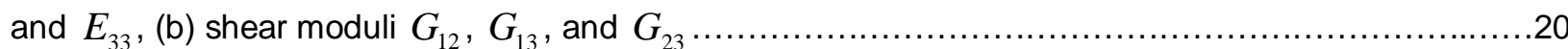

Figure 11. Predicted elastic properties for the glass/PEEK composites as a function of fiber volume fraction $v$ and fiber length distribution represented by the weight-average length: (a) Moduli $E_{11}, E_{22}$,

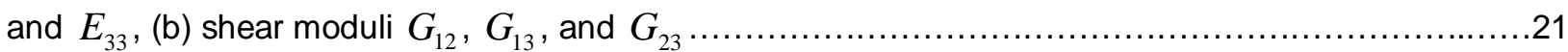

Figure 12. Comparison of the elastic moduli $E_{11}$ for three injection-molded composites: glass/PP, glass/PA 6,6, and glass/PEEK. 30\% fiber volume fraction and the FLD-3 in Figure 7 were used in the

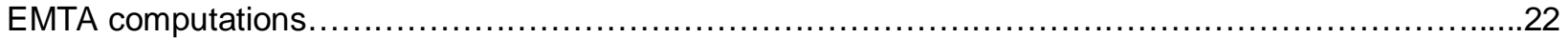
Figure 13. Assumed fiber length distributions for the EMTA calculation of the elastic properties for

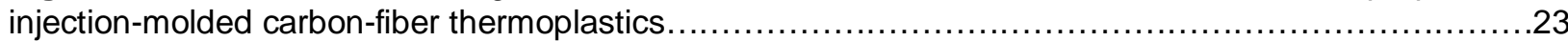
Figure 14. Schematic picture describing the computational procedure to predict the elastic properties of a

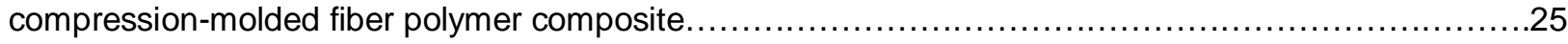

Figure 15. Predicted elastic moduli $E_{11}=E_{22}$ for compression-molded chopped fiber composites.......26

Figure 16. Predicted elastic modulus $G_{12}$ for compression-molded chopped fiber composites............27 


\section{List of Tables}

Table 1. Elastic properties of the glass fibers and of the resins used in the EMTA calculations..........17

Table 2. Elastic properties of the carbon fibers used in the EMTA calculations [8] ......................22

Table 3. EMTA elastic property results for the injection-molded carbon/PP, carbon/PA 6,6, and carbon/PEEK. $20 \%$ fiber volume fraction was used in the computations..................................23

Table 4. EMTA elastic property results for the injection-molded carbon/PP, carbon/PA 6,6, and carbon/PEEK. 30\% fiber volume fraction was used in the computations................................24

Table 5. Elastic properties for the carbon fiber tow and glass fiber tow predicted by EMTA ..............25 


\section{Introduction}

Fiber-reinforced polymer composite materials are finding increasing applications underwater where their low specific weight, high mechanical performance, and excellent corrosion resistance offer significant advantages over metallic materials. For instance, they have been used in deep sea applications, boat structures, offshore drilling platforms, etc. In view of these applications, there is an interest to use fiber polymer composites for hydropower systems. Applying polymer composites to today's hydropower systems is still in the proof of concept stage where design, component processability, and cost effective manufacturing have not yet been addressed. An important feature that would make the applications of polymer composites in hydropower systems possible resides in the possibility to tailor these materials to achieve the desired mechanical performance. This can be performed by appropriately selecting the fiber/resin material combinations, fiber and resin features, and processing methods. In addition, processing parameters can be optimized to improve part quality and mechanical performance.

As a first step in the composite material design for hydropower applications, this project task has used a predictive modeling tool named EMTA (Copyright (C) Battelle 2010) developed at PNNL to evaluate the elastic properties of various fiber-reinforced polymer composites for their potential applications in hydropower systems. EMTA implements the standard and improved Eshelby-Mori-Tanaka approaches to compute the composite thermoelastic properties accounting for the constituents' properties and features such as the thermoelastic properties of the fibers and of the resin, fiber length and orientation distributions, fiber curvature, and initial micro-defects [1-8]. The evaluation will consider both glass and carbon fibers embedded in a thermoplastic or a thermoset matrix.

The structure of this report is as follows. After the Introduction, processing methods to form various composite systems will be discussed to apprehend the composite microstructure and the corresponding values of elastic properties that could be obtained when using a given method to make the composite component or part (Section 2). In Section 3, a summary of the key micromechanical models behind EMTA is presented. EMTA will then be applied to compute the elastic properties of the as-formed composites as the functions of the constituents' properties, fiber volume fraction, fiber length, and fiber orientation distributions (Section 4). The continuous fiber composite is regarded as a particular case of a discontinuous fiber composite whose fiber aspect ratio is extremely large and fiber orientation is known in advance. The conclusions (Section 5) will provide comments on the type of composite and the associated elastic stiffness range which would be appropriate for a given hydropower system.

In the underwater environment, polymer composite structures are subjected to moisture absorption, high pressure, and fatigue wave loading. In general, moisture absorption causes a reduction of the composite stiffness and strength that leads to premature cracking [9-10]. It has been recognized that hydrostatic pressure affects the behavior of polymers. The main effect is an increase in modulus and yield stress [11]. However, tensile strength tends to decrease with increasing pressure and this has been related to increased matrix yield stress and reduced stiffness. Fatigue wave loading causes cracking in the polymer matrix that can engender other catastrophic failure mechanisms which are delamination and fiber rupture. If the fibers are discontinuous, matrix cracking is also one of the main failure mechanisms. It is important to 
emphasize that the evaluation of the composite elastic stiffness is only the first step in the material design. Nonlinear behaviors of the composite due to damage and inelastic deformations under loading and environmental conditions must be addressed before the composite could be used with confidence in hydropower systems.

\section{Processing Methods for Discontinuous Fiber Polymer Composites}

In this section, injection-molding and compression molding of fiber-filled polymers are reviewed as these highly-automated processing methods are commonly used to quickly produce cost-effective semi-structural or structural discontinuous fiber polymer composite parts. This gives them an economic advantage over the processing methods for continuous-fiber composites, which are still slow and labor-intensive. Nevertheless, the mechanical properties of a discontinuous fiber composite are not as good as those for a composite with continuous reinforcing fibers. Therefore, it is important to assess the mechanical properties of discontinuous fiber composites to determine their potential applications in hydropower systems. As mentioned in the Introduction, processing methods and parameters play an important role in determining the composite properties.

\subsection{Injection Molding of Fiber-filled Thermoplastics}

Injection molding of short- or long-fiber filled thermoplastics is probably the most attractive and economic method for producing complex composite parts of relatively good stiffness. During injection molding, thermoplastic pellets (fiber-polymer compounds) containing fibers are fed into a heated barrel where the polymer softens and becomes a viscous melt. In the barrel, which contains the reciprocating screw, the thermoplastic is gradually melted by a combination of shear heating and heat conduction from the barrel. Supplementary heaters help maintain a constant temperature in the barrel. As the volume of the melt in front of the screw increases, it forces the screw to the rear of the barrel. Hence, it is necessary to apply a pressure (back pressure) to the back end of the screw. Increasing the back pressure will increase the mechanical work on the fiber/polymer mixture. When enough material has accumulated in front of the screw, the screw advances and drives the polymer/fiber mixture into the mold. After the composite has solidified, the mold is opened and the part is ejected [12-13]. An example of an injection molding machine is shown in Figure 1.

Under the screw action, fibers undergo excessive bending and shearing that break them. As a consequence, their lengths degrade importantly before they enter the mold cavity. There is additional length attrition in the mold due the shearing of the fibers that occurs during the flow of the fiber suspension. The length attrition becomes more important when the fiber volume fraction is high, therefore, to conserve fiber length to achieve good mechanical properties, fiber volume fractions should not be too high. Current injection molding technologies can make composite parts with 30\% fiber volume fraction. The screw design can be optimized to reduce fiber length degradation. In addition, process parameters such as the injection speed, back pressure, mold and melt temperatures should also be optimized to better conserve fiber length. For instance, low back pressure and slow fill conditions could reduce the length attrition. 


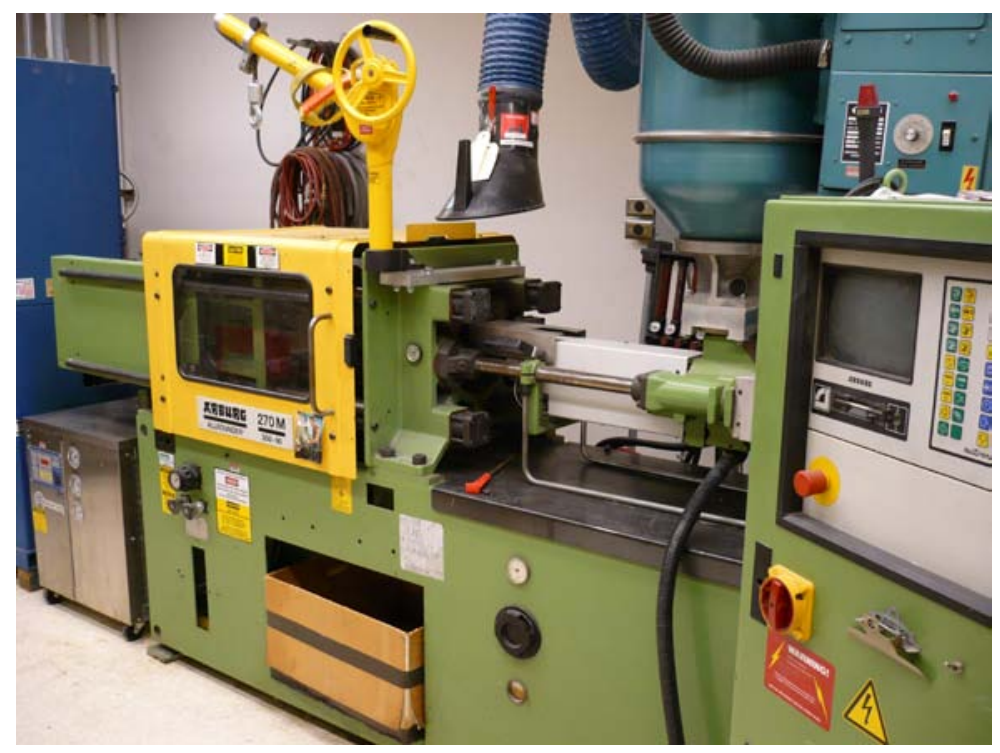

Figure 1. PNNL's injection molding machine.

\subsection{Compression Molding of Fiber Preforms}

Compression molding encompasses several technologies that are related to compression molding of sheet molding compounds (SMC), compression molding of structural molding compounds, and compression molding of fiber thermoplastics. One common feature of all the compression molding processes is the use of a "preform" material or "charge" that is pressed to shape and cured during the molding operation. Currently, compression molding is the most versatile way to incorporate random chopped fibers into a structural composite. Depending on component's shape and charge, compression molding may involve regions with fiber suspension flow (somewhat similar to what observed in injection molding) that tends to orient fibers in the flow direction [14]. It may also induce very little flow, and the fibers remain practically twodimensionally random. Figure 2 shows a schematic of the compression molding operation.

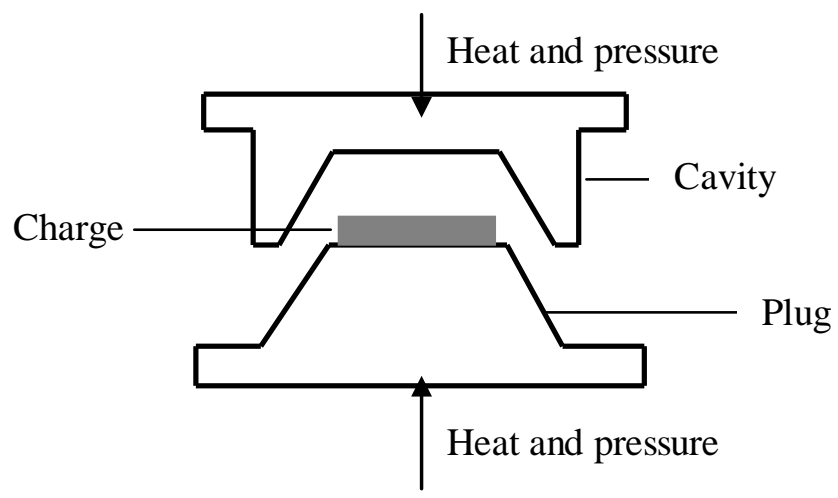

Figure 2. Schematic of a compression molding operation. 
A typical compression molding process cycle includes placing the preforms made of chopped fibers in a chemically thickened thermoset resin into a heated mold, and then closing the mold under pressure for about 1 to 3 minutes to cure the material. The charge occupies an important space in the mold. Once the mold is closed, the material flows to fill the remaining cavity. An example of a compression molding machine is presented in Figure 3.

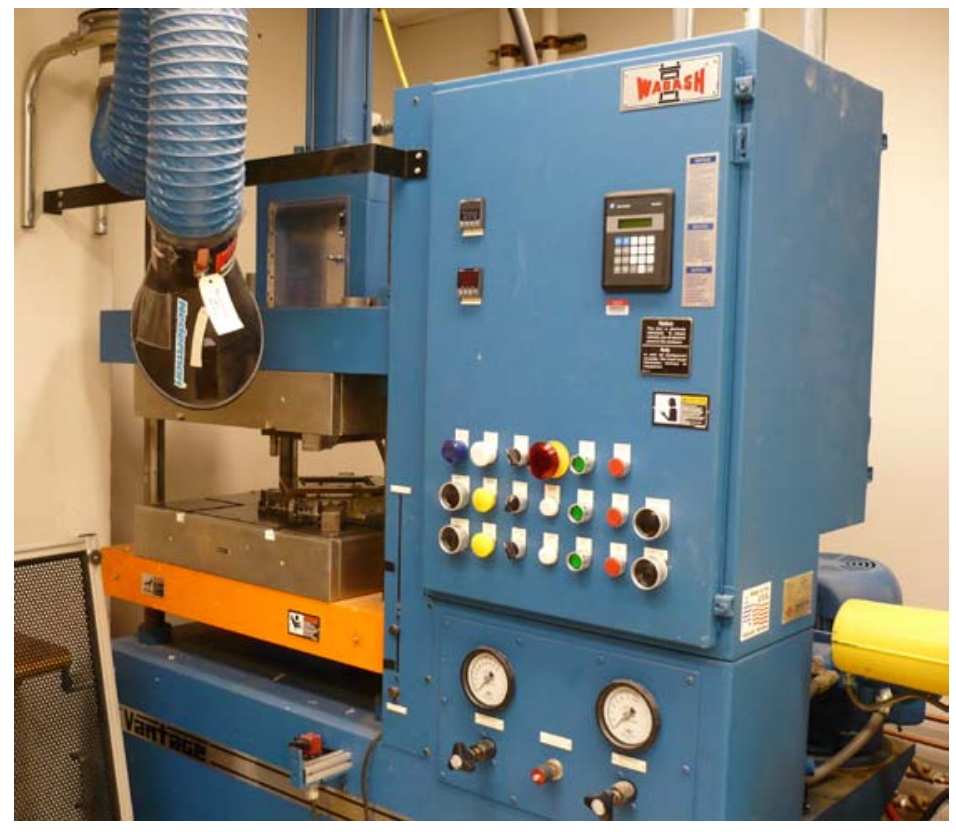

Figure 3. PNNL's compression molding machine.

There are two important advantages of compression molding over injection molding. These are the conservation of fiber length and much higher fiber volume fraction that can be achieved in a compression-molded composite. In fact, very little fiber length attrition occurs during compression molding. The as-formed composite possesses practically the same fiber length as the preform. Fiber lengths can be as long as $50 \mathrm{~mm}$, and high fiber volume fractions up to $60 \%$ can be achieved in a compression-molded composite. These advantages combined enable much better mechanical properties to be achieved for compression-molded materials compared to injection-molded materials. However, the injection molding process has the advantages of more readily being automated and of producing finer part details that cannot be obtained by compression molding.

\section{The Eshelby-Mori-Tanaka Approach and EMTA Software}

A large body of literature exists addressing the prediction of elastic properties of non-dilute aligned fiber composites. In particular, there is a family of models that has evolved from a proposal initially made by Mori and Tanaka [2]. To date, the Eshelby's equivalent inclusion method [1] combined with a Mori-Tanaka type model presents one of the most efficient and accurate approaches for the prediction of the composite stiffness $[4,6]$. However, it has been 
shown that the standard Eshelby-Mori-Tanaka model is accurate for fiber volume fractions less than $20 \%$ to $30 \%$. For higher fiber volume fractions, formulations $[8,15]$ have been developed to improve the standard model. This section summarizes both the standard and improved models that have been implemented in EMTA. EMTA has previously been validated for both injectionmolded and compression-molded fiber polymer composites [6, 8].

\subsection{The Standard Eshelby-Mori-Tanaka Model}

The Eshelby-Mori-Tanaka (EMT) model uses the Eshelby's equivalent inclusion method that is schematically described in Figure 4. The body that contains an inclusion (b) is replaced by a homogeneous body (a) of the same matrix material. Both bodies are subject to the same strain field $\boldsymbol{\varepsilon}_{0}$. In order that the homogeneous body has the same response as the inhomogeneous body, some transformation strain or eigenstrain $\varepsilon^{*}$ must be introduced such that the perturbation strain that is due to the presence of the inclusion is given in terms of the eigenstrain as

$$
\boldsymbol{\varepsilon}^{\mathrm{pt}}=\mathrm{S} \boldsymbol{\varepsilon}^{*}
$$

where $\mathbf{S}$ is the Eshelby tensor that depends on the inclusion aspect ratio and the matrix elastic properties. Expressions of $\mathbf{S}$ for different inclusion shapes are given by Mura [16]. As a result, the stress in the inclusion is given by

$$
\boldsymbol{\sigma}_{\mathrm{f}}=\mathrm{C}_{\mathrm{f}}\left(\boldsymbol{\varepsilon}_{0}+\overline{\boldsymbol{\varepsilon}}+\boldsymbol{\varepsilon}^{\mathrm{pt}}\right)=\mathrm{C}_{\mathrm{m}}\left(\boldsymbol{\varepsilon}_{0}+\overline{\boldsymbol{\varepsilon}}+\boldsymbol{\varepsilon}^{\mathrm{pt}}-\boldsymbol{\varepsilon}^{*}\right)
$$

where $\mathbf{C}_{\mathrm{f}}$ and $\mathbf{C}_{\mathrm{m}}$ are the stiffness tensors of the inclusion and the matrix material, respectively. $\overline{\boldsymbol{\varepsilon}}$ is the strain in the matrix. Using the equivalent inclusion method expressed by Equation (2) for a non-dilute composite, and considering that the perturbation stress over the representative volume element must vanish, leads to

$$
\mathbf{C}=\mathbf{C}_{\mathrm{f}}+f\left(\mathbf{C}_{\mathrm{f}}-\mathbf{C}_{\mathrm{m}}\right) \mathbf{A}
$$

where $f$ is the fiber volume fraction, and $\mathbf{A}$ is the strain concentration tensor given by

with

$$
\mathbf{A}=\mathbf{T}[(1-f) \mathbf{I}+f \mathbf{T}]^{-1}
$$

$$
\mathbf{T}=\left[\mathbf{I}+\mathbf{S C}_{\mathrm{m}}^{-1}\left(\mathbf{C}_{\mathrm{f}}-\mathbf{C}_{\mathrm{m}}\right)\right]^{-1}
$$

In EMTA, the Eshelby-Mori-Tanaka formulation by Chen and Cheng [17] has been implemented, and Equation (3) is equivalent to

where

$$
\mathbf{C}=\mathbf{C}_{\mathrm{m}}\left[\mathbf{I}+f \mathbf{B}(\mathbf{I}+f \mathbf{E})^{-1}\right]^{-1}
$$

$$
\mathbf{B}=\left[\mathbf{C}_{\mathrm{m}}(\mathbf{S}-\mathbf{I})-\mathbf{C}_{\mathrm{f}} \mathbf{S}\right]^{-1}\left(\mathbf{C}_{\mathrm{f}}-\mathbf{C}_{\mathrm{m}}\right) \text { and } \mathbf{E}=(\mathbf{S}-\mathbf{I}) \mathbf{B}
$$




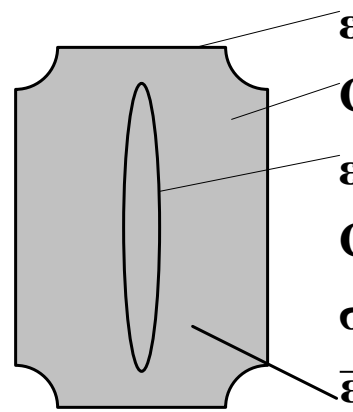

(a)

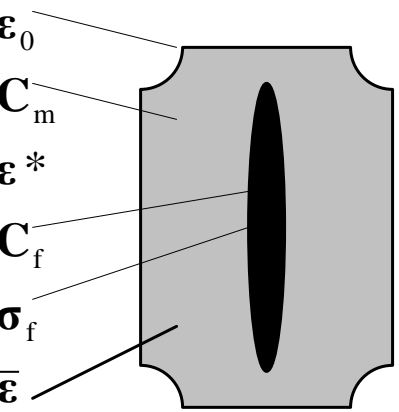

(b)

Figure 4. Eshelby's equivalent inclusion method.

\subsection{An Eshelby-Mori-Tanaka Model for Concentrated Composites}

In order to extend the EMT model to concentrated composites, the concentration tensor needs to be calculated in a way that incorporates the fiber-fiber interaction. Intuitively, in high fiber concentrations, a fiber does not see the matrix material but a composite medium around it. Therefore, a simple idea $[15,8]$ is to define an equivalent isotropic composite medium that surrounds the fiber. This medium has a fiber volume fraction $f^{\prime}$ between 0 and $f$. If $f^{\prime}=0$, we recover the standard EMT model, and for any value of $f^{\prime}$ between 0 and $f$, improved EMT solutions for concentrated composites are obtained. Hence, $f^{\prime}$ also serves as a fitting parameter to better correlate the predicted properties with the experimental values. The EMT solution for concentrated composites is given by

$$
\mathbf{C}=\mathbf{C}_{\mathrm{f}}+f\left(\mathbf{C}_{\mathrm{f}}-\mathbf{C}_{\mathrm{m}}\right) \mathbf{A} *
$$

where $\mathbf{A}^{*}$ is the strain concentration tensor of the equivalent medium given by:

with

$$
\mathbf{A}^{*}=\mathbf{T}^{*}\left[(1-f) \mathbf{I}+f \mathbf{T}^{*}\right]^{-1}
$$

$$
\mathbf{T}^{*}=\left[\mathbf{I}+\mathbf{S}_{\mathrm{eq}} \mathbf{C}_{\mathrm{eq}}^{-1}\left(\mathbf{C}_{\mathrm{f}}-\mathbf{C}_{\mathrm{eq}}\right)\right]^{-1}
$$

where $\mathbf{C}_{\text {eq }}$ and $\mathbf{S}_{\text {eq }}$ are the stiffness and Eshelby's tensor of the equivalent medium, respectively. In EMTA, the equivalent isotropic composite medium is defined considering $f^{\prime}=f / 2$, and the fibers of this medium have the same aspect ratio as the actual composite but are completely randomly oriented to guarantee its global isotropic character.

\subsection{Solution for Misoriented Fiber Composites}


The standard and EMT models for high concentrations presented in the previous sections allow the computation of the elastic properties for an aligned fiber composite. For a composite material containing fibers whose orientations obey some distribution, it is necessary to account for the fiber orientation distribution in the prediction of the composite properties. The elastic stiffness of a composite having a given fiber orientation distribution is computed using the orientation averaging method [18]:

$$
\begin{aligned}
\bar{C}_{i j k l}= & B_{1} A_{i j k l}+B_{2}\left(A_{i j} \delta_{k l}+A_{k l} \delta_{i j}\right)+B_{3}\left(A_{i k} \delta_{j l}+A_{i l} \delta_{j k}+A_{j l} \delta_{i k}+A_{j k} \delta_{i l}\right) \\
& +B_{4} \delta_{i j} \delta_{k l}+B_{5}\left(\delta_{i k} \delta_{j l}+\delta_{i l} \delta_{j k}\right)
\end{aligned}
$$

where $A_{i j}$ and $A_{i j k l}$ are the components of the second- and fourth-order orientation tensors, respectively, and the coefficient $B_{i}(i=1, \ldots, 5)$ are related to the components of the stiffness tensor of the aligned fiber composite given in Equation (6) or (8). $\delta_{i j}$ denotes the components of the identity tensor. The expressions of $B_{i}$ can be found in [18]. The orientation tensors $A_{i j}$ and $A_{i j k l}$ are theoretically given in terms of the orientation vector that is parallel to the fiber axis (Figure 5). As it is not possible or necessary to quantify the orientation of each fiber, the fiber orientation is defined over a volume domain, thus it represents a volume average.

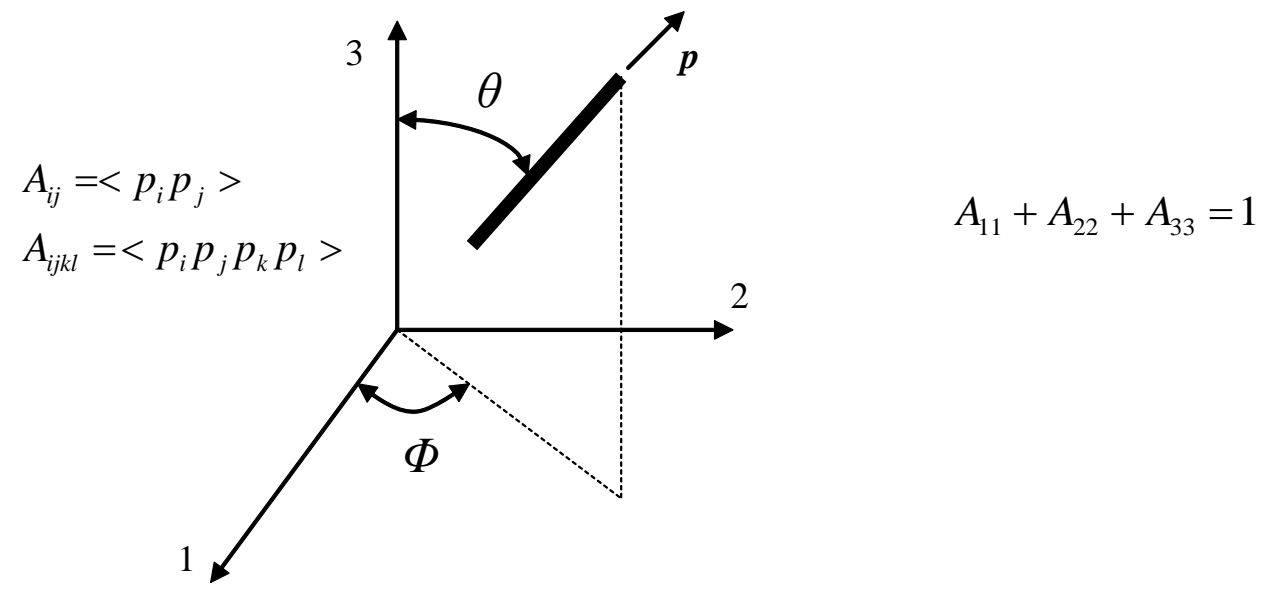

Figure 5. Theoretical definitions of the second- and fourth-order orientation tensors in terms of the orientation vector components.

\subsection{Solution for Composites with Fiber Length Distribution}

If the composite contains a fiber length distribution, its stiffness is computed as [6]:

$$
C_{i j k l}^{*}=\frac{\int_{0}^{\infty} C_{i j k l}(l / d) p(l) d l}{\int_{0}^{\infty} p(l) d l}
$$


where $C_{i j k l}$ is the stiffness matrix of the unidirectional composite having the fiber aspect ratio $l / d$, where $l$ is the fiber length and $d$ is the fiber diameter. $C_{i j k l}$ is given by Equation (6) or Equation (8). $p(l)$ is the probability density function (PDF) that expresses the fiber length distribution (FLD) in terms of number or weight of fibers versus fiber length. Introducing Equation (12) to Equation (11), we obtain the stiffness tensor of a composite containing a fiber orientation distribution in addition to a fiber length distribution.

Bending of long fibers causes fiber curvature in the composite. In addition, microdefects such as microvoids and partial fiber/matrix decohesion can exist after processing. The EMT based models accounting for these features have been developed and implemented in EMTA [5, 7], these models have not been used in this report as we assume straight fibers, perfect fiber/matrix interfaces and non-existence of microvoids for the estimate of the composite stiffness.

\section{Numerical Applications}

\subsection{EMTA Computation of Elastic Properties for Injection-Molded Fiber Thermoplastics}
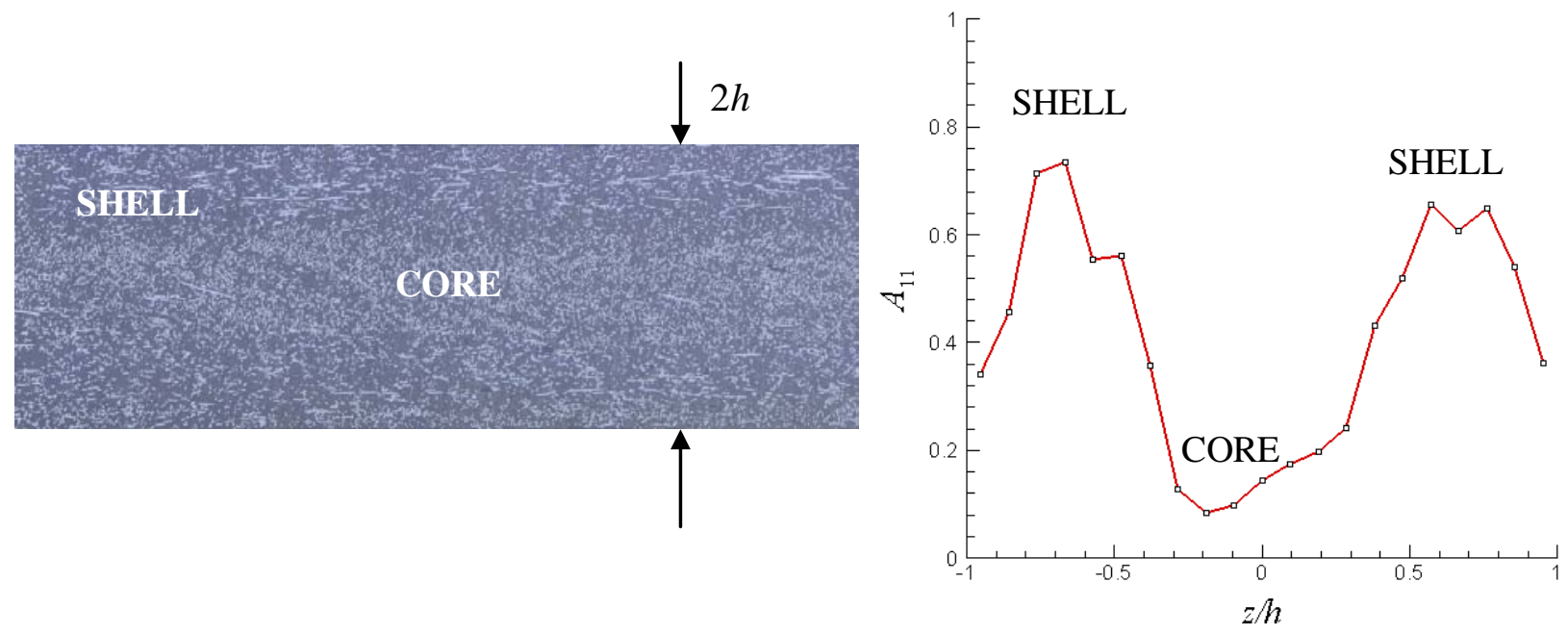

Figure 6. (a) A micrograph of a cross section of an injection-molded fiber material showing the orientation layer structure, and (b) Typical through-thickness variation of the $A_{11}$ component in a long-fiberthermoplastic (LFT) material.

During injection molding, fibers enter the mold cavity under high pressure and high velocity. The flow of the fiber suspension and the interaction among fibers induce a fiber orientation structure in the molded composite. The fiber orientation structure involves the skin, shell, and core layers which are typical to injection-molded short-/long-fiber thermoplastics (Figure 6.). At the skin layers (outer layers), the fiber orientation is generally two-dimensionally random with 
$A_{11} \approx A_{22} \approx 0.5$. The fibers are rather aligned with the flow direction in the shells with higher values of $A_{11}$ (from about 0.65 to 0.8 ) and lower values of $A_{22}$ (from about 0.3 to 0.2 ) while they are rather cross-flow oriented in the core with higher values of $A_{22}$ (from about 0.7 to 0.9 ). Although both injection-molded short- and long-fiber thermoplastic composites possess a skin/shell/core orientation layer structure, there are significant differences in the fiber orientation structure found in a short-fiber thermoplastic (SFT) compared to the orientation structure observed in a long-fiber thermoplastic (LFT) composite. Fibers are considered to be long if their lengths are greater than $1 \mathrm{~mm}$. In a SFT (fiber length much less than $1 \mathrm{~mm}$ ), the core is much narrower than the core of a long-fiber thermoplastic but the shells are larger and more aligned with the flow direction. For instance, values of $A_{11}$ for the SFT shell layers can attain 0.8 while those for the LFT shells are about 0.65 to 0.7 .

In addition to fiber orientation distribution, the microstructures of injection-molded SFTs and LFTs possess a fiber length distribution (FLD) resulting mainly from the breakage of fibers in the screw and nozzle. Fiber length attrition is so important that starting from a 13-mm pellet length, the average residual length is less than $1 \mathrm{~mm}$ for SFTs and about 2 and $3 \mathrm{~mm}$ for LFTs.

In view of fiber orientation and length distributions that determine the properties of SFTs and LFTs, the prediction of any properties for these materials must account for these distributions. In the following, for a general estimate of the elastic properties of injection-molded materials for potential applications in hydropower systems, we rather look for long-fiber materials for better mechanical performance. Figure 7 shows a typical fiber orientation distribution for an injectionmolded LFT material. This fiber orientation distribution is assumed in the EMTA calculations of the elastic properties for all the injection-molded LFTs.
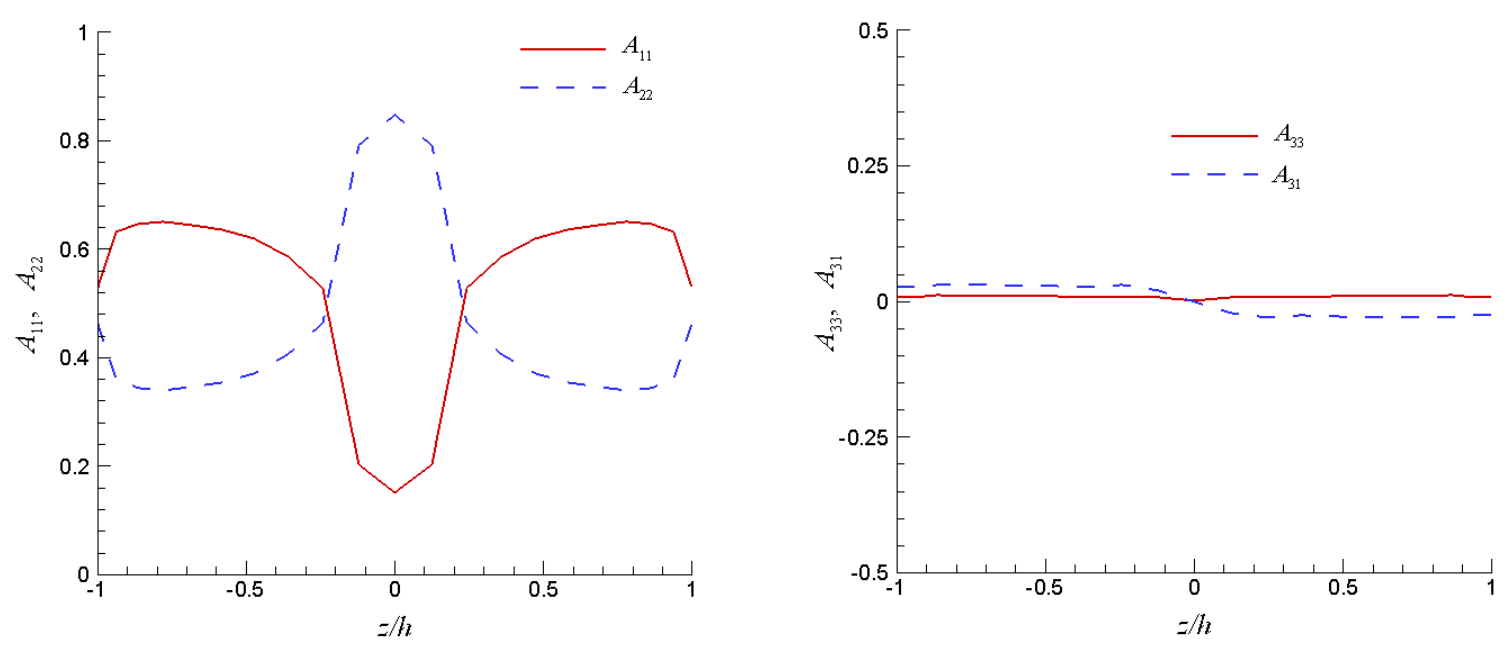

Figure 7. Assumed fiber orientation distribution for the EMTA calculation of the elastic properties for injection-molded LFTs. 
As our goal is to improve the composite stiffness by conserving fiber length (minimizing fiber length degradation), we consider a series of FLDs as depicted in Figure 8 that expresses the FLDs for weight of fibers versus fiber length. On this figure, FLD-1 represents the FLD for a reference long-glass fiber thermoplastic material. FLD-1 is the FLD that is achievable in practice using the conventional injection-molding technologies. FLD-2, FLD-3, FLD-4, and FLD-5 are the assumed FLDs that are sought to enhance the composite elastic properties.

The elastic properties of the constituent materials (glass fibers and resin matrices) used in the EMTA calculations are given in Table 1. The thermoplastic resins considered are polypropylene (PP), polyamide 6,6 (PA 6,6), and poly-ether-ether-ketone (PEEK).

\begin{tabular}{|c|c|c|c|c|}
\hline & Glass Fiber & PP [6] & PA 6,6 [19] & PEEK [20] \\
\hline Elastic Modulus (MPa) & 72300 & 1500 & 2900 & 4100 \\
\hline Poisson's Ratio & 0.2 & 0.4 & 0.35 & 0.38 \\
\hline
\end{tabular}

Table 1. Elastic properties of the glass fibers and of the resins used in the EMTA calculations.

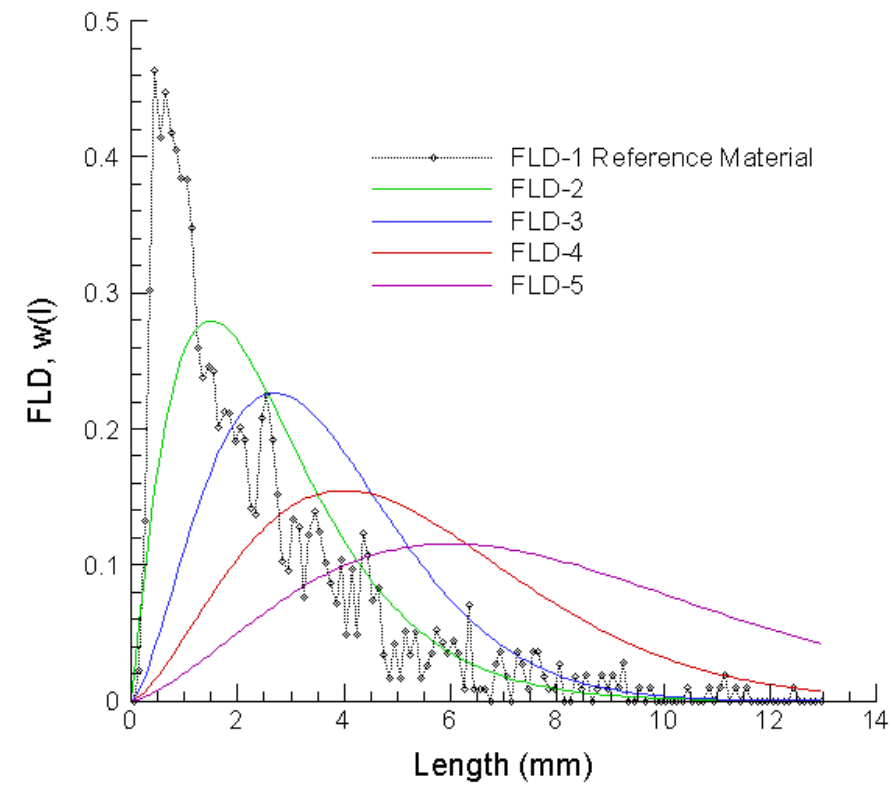

Figure 8. Assumed fiber length distributions for the EMTA calculation of the elastic properties for injection-molded long-glass-fiber thermoplastics.

\subsubsection{Results for Glass/PP}


Figure 9 presents the EMTA results for the elastic properties of the injection-molded glass/PP composites having the fiber volume fraction of 0.2 and 0.3 . The calculations used the fiber orientation and length distributions given in Figures 6 and 7. Figure 8 shows that improving fiber length by using the distributions from FLD-1 to FLD-3 results in a noticeable gain in the elastic properties for the composite. However, beyond FLD-3, there is little improvement in elastic properties. This is because FLD-3 has already achieved a "good" fiber length to attain the limit of stiffness. On the other hand, increasing fiber volume fraction from 0.2 to 0.3 leads to important increases in elastic properties (about $40 \%$ increase in moduli $E_{11}$ and $E_{22}$, and $23 \%$ increase in $E_{33}$, and $41 \%$ increase in the shear modulus $G_{12}$ ).

\subsubsection{Results for Glass/PA6,6}

Figure 10 presents the EMTA results for the elastic properties of the injection-molded glass/PA 6,6 composites having the fiber volume fraction of 0.2 and 0.3 . Compared to the results for glass/PP, the use of a nylon matrix has allowed the composite elastic properties to be theoretically significantly improved. However, caution should be exercised while applying PA6,6 to hydropower systems as their properties can degrade importantly due to moisture absorption. As observed for the glass/PP composites, noticeable increase in the composite elastic properties is achieved by increasing fiber lengths from FLD-1 to FLD-3, however, beyond FLD3 , little improvement happens as the limit of stiffness is practically attained. On the other hand, increasing fiber volume fraction from 0.2 to 0.3 results in important increases in elastic

properties (about 35\% increase in moduli $E_{11}, E_{22}$, and $21 \%$ increase in $E_{33}$, and $36 \%$ increase in the shear modulus $G_{12}$ ).

\subsubsection{Results for Glass/PEEK}

The EMTA results for the elastic properties of the injection-molded glass/PEEK composites having the fiber volume fraction of 0.2 and 0.3 are given in Figure 11. As observed in the previous composites, increasing fiber lengths from FLD-3 to FLD-5 has little effect on the composite elastic properties because FLD-3 has achieved a "sufficient" length for reaching the stiffness limit. However, increasing the fiber volume fraction from 0.2 to 0.3 results in more important increase in elastic properties (about 32\% increase in moduli $E_{11}, E_{22}$, and 22\% increase in $E_{33}$, and $34 \%$ increase in the shear modulus $G_{12}$ ). It is noted that the higher elastic modulus of the PEEK matrix allows significantly higher elastic properties for the glass/PEEK composites in comparison to the elastic properties for glass/PP and glass/PA 6,6 composites. This has been illustrated on Figure 12 for the main modulus $E_{11}$. 
Long-glass-fiber/polypropylene

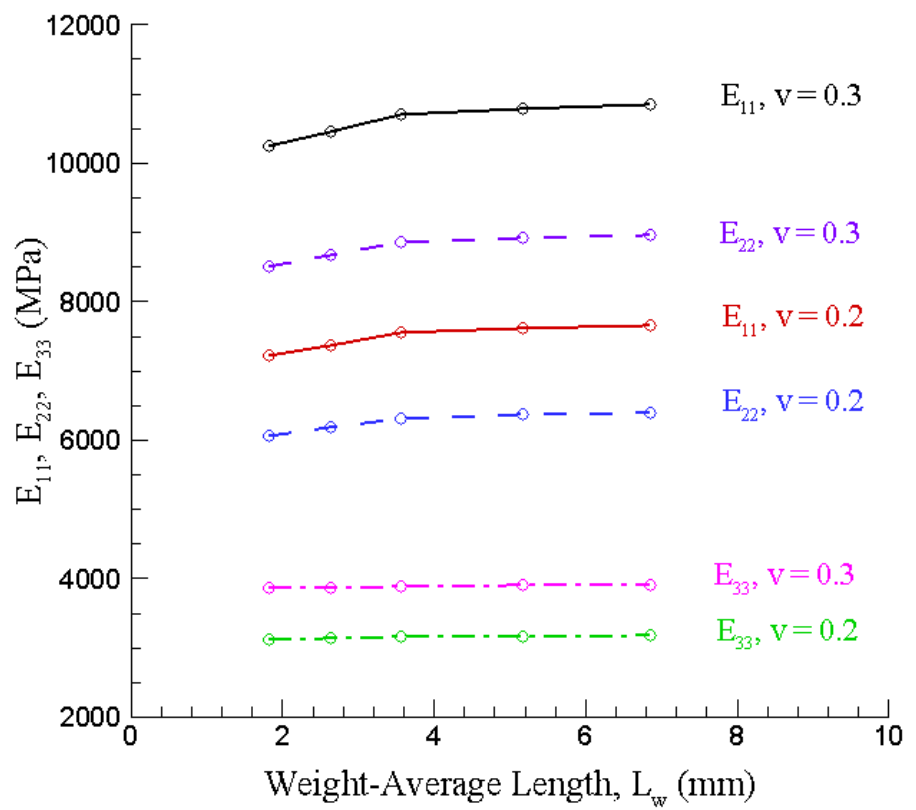

(a)

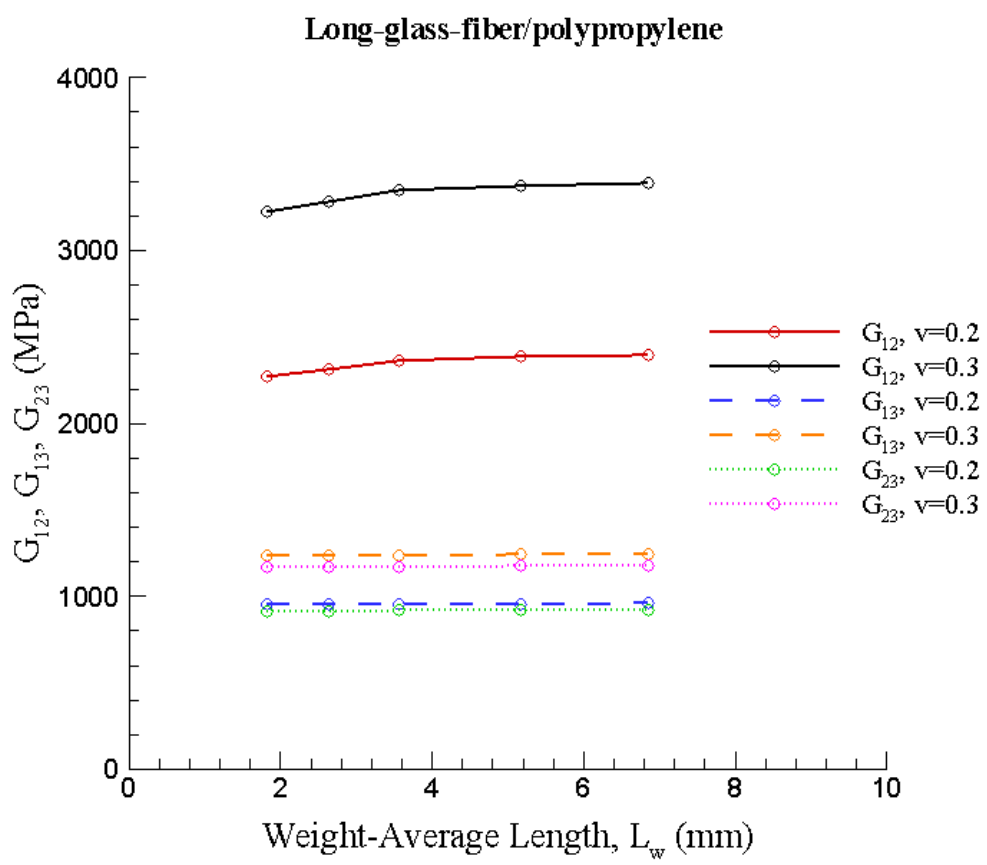

(b)

Figure 9. Predicted elastic properties for the glass/PP composites as a function of fiber volume fraction $v$ and fiber length distribution represented by the weight-average length: (a) Moduli $E_{11}, E_{22}$, and $E_{33}$, (b) shear moduli $G_{12}, G_{13}$, and $G_{23}$. 


\section{Long-glass-fiber/PA6,6}

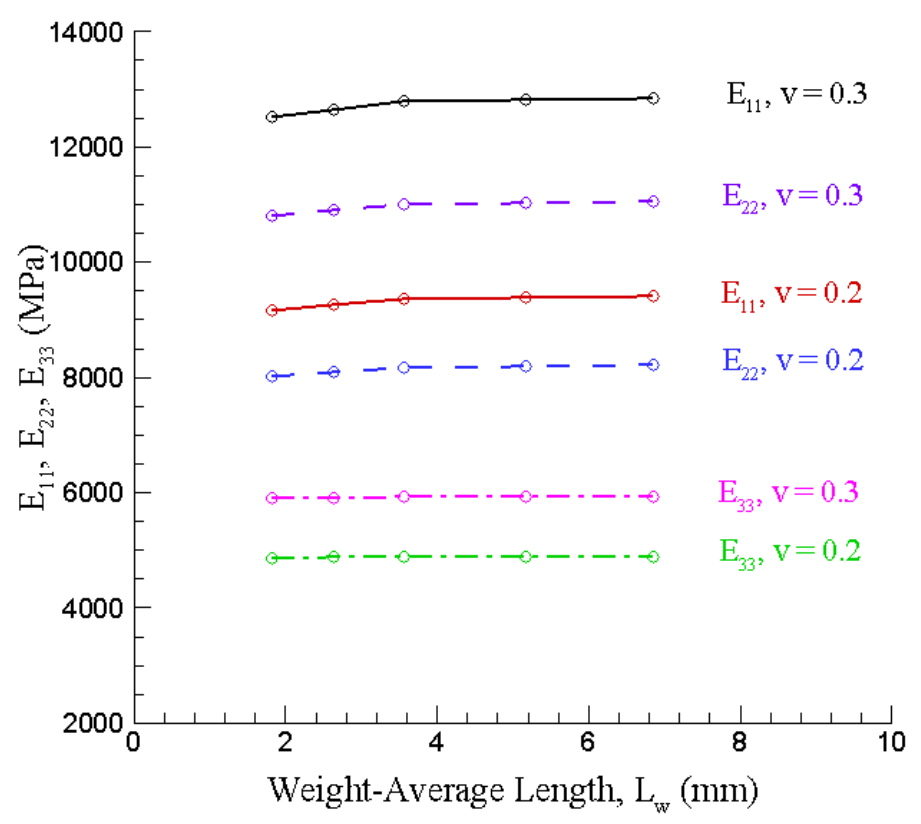

(a)

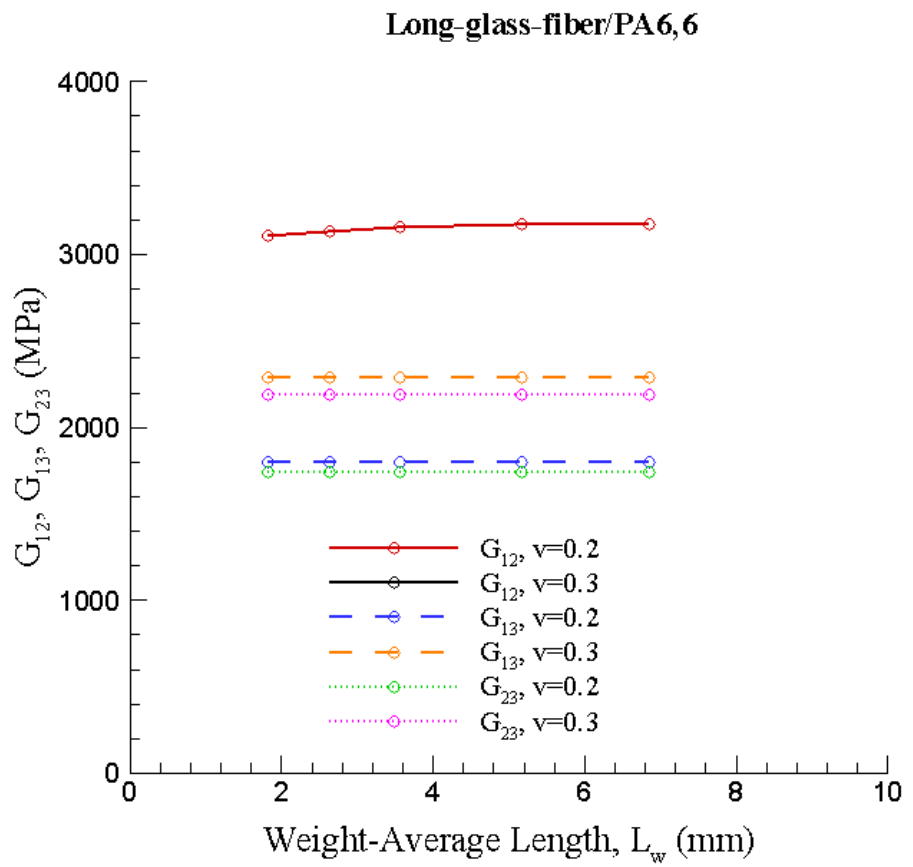

(b)

Figure 10. Predicted elastic properties for the glass/PA 6,6 composites as a function of fiber volume fraction $v$ and fiber length distribution represented by the weight-average length: (a) Moduli $E_{11}, E_{22}$, and $E_{33}$, (b) shear moduli $G_{12}, G_{13}$, and $G_{23}$. 
Long-glass-fiber/PEEK

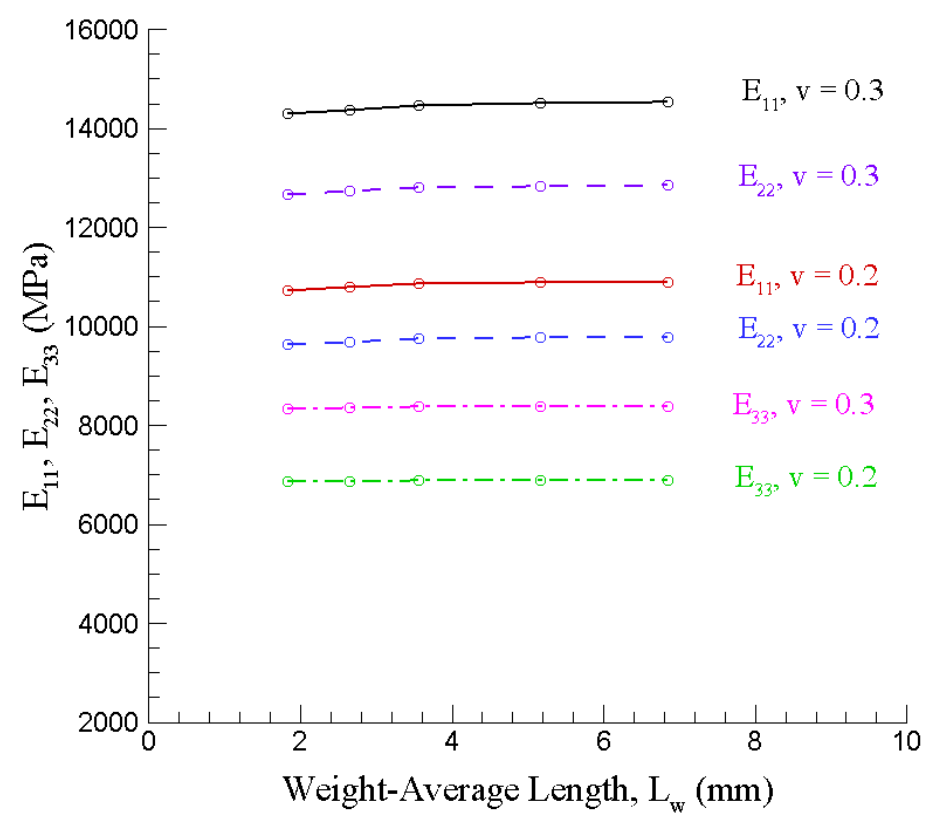

(a)

Long-glass-fiber/PEEK

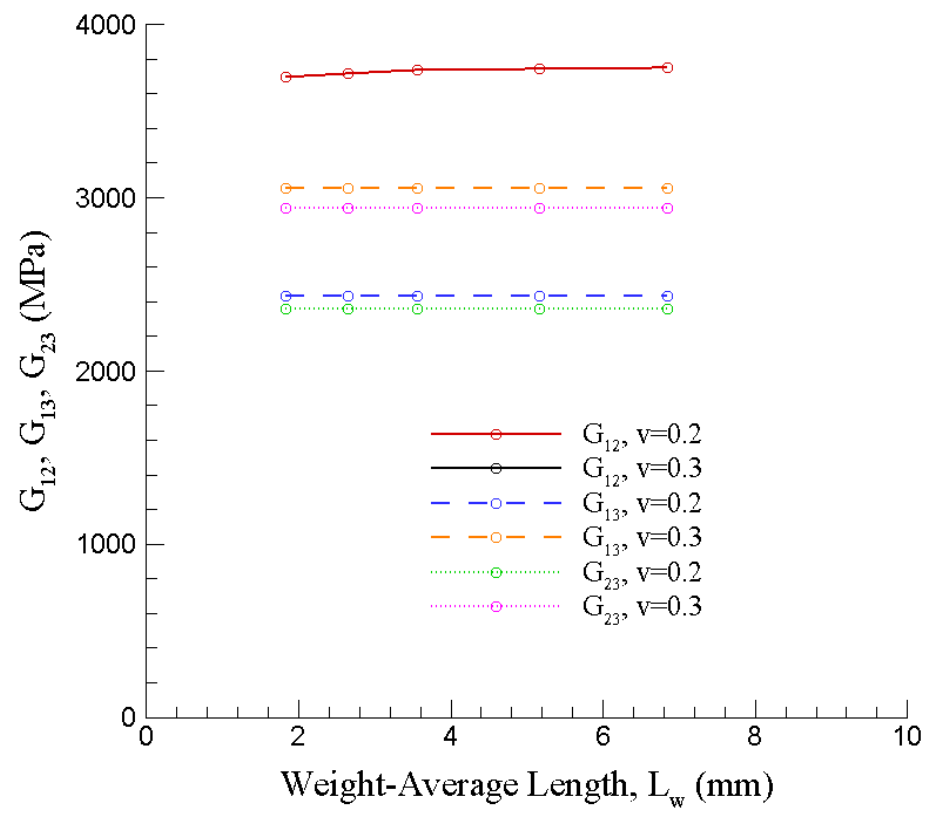

(b)

Figure 11. Predicted elastic properties for the glass/PEEK composites as a function of fiber volume fraction $v$ and fiber length distribution represented by the weight-average length: (a) Moduli $E_{11}, E_{22}$, and $E_{33}$, (b) shear moduli $G_{12}, G_{13}$, and $G_{23}$. 


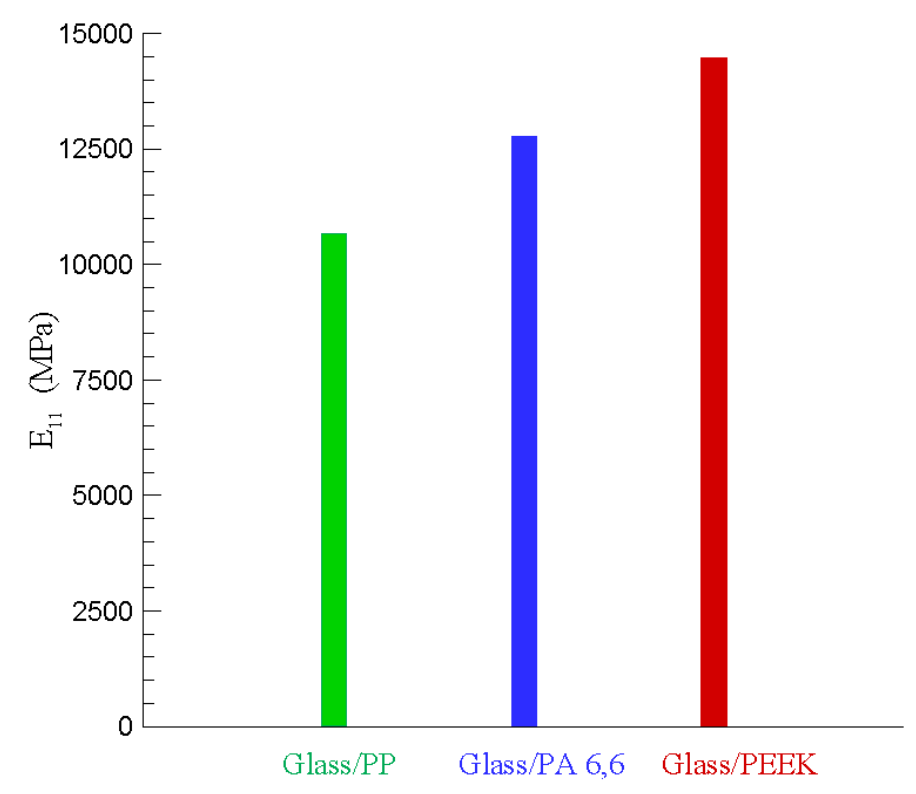

Figure 12. Comparison of the elastic moduli $E_{11}$ for three injection-molded composites: glass/PP, glass/PA 6,6, and glass/PEEK. 30\% fiber volume fraction and the FLD-3 in Figure 7 were used in the EMTA computations.

\subsubsection{Results for Carbon/PP, Carbon/PA 6,6, and Carbon/PEEK}

Current injection molding technologies are still unable to injection mold truly long-carbonfiber thermoplastic composites. There are issues related to the carbon fiber coating to ensure a good adhesion with some thermoplastic resins like polypropylene. In addition, long carbon fibers are not well dispersed during injection molding resulting in poor fiber coverage in the molded composites. Poor fiber/matrix bonding and poor fiber dispersion result in low mechanical properties although carbon fibers have excellent mechanical properties. Because of these reasons, it is not realistic to consider the FLDs presented in Figure 5 but rather the FLD presented in Figure 13 for a shorter fiber system. The properties of carbon fibers used in the EMTA computation are given in Table 2 where $E_{\mathrm{L}}, E_{\mathrm{T}}$, and $G_{\mathrm{LT}}$ denote the longitudinal, transverse, and longitudinal shear moduli, respectively. $v_{\mathrm{LT}}$ and $v_{\mathrm{TT}}$ are the longitudinal and transverse Poisson's ratios.

\begin{tabular}{|c|c|c|c|c|}
\hline$E_{\mathrm{L}}(\mathrm{MPa})$ & $E_{\mathrm{T}}(\mathrm{MPa})$ & $G_{\mathrm{LT}}(\mathrm{MPa})$ & $v_{\mathrm{LT}}$ & $v_{\mathrm{TT}}$ \\
\hline 231000 & 13800 & 12400 & 0.2 & 0.25 \\
\hline
\end{tabular}

Table 2. Elastic properties of the carbon fibers used in the EMTA calculations [8]. 


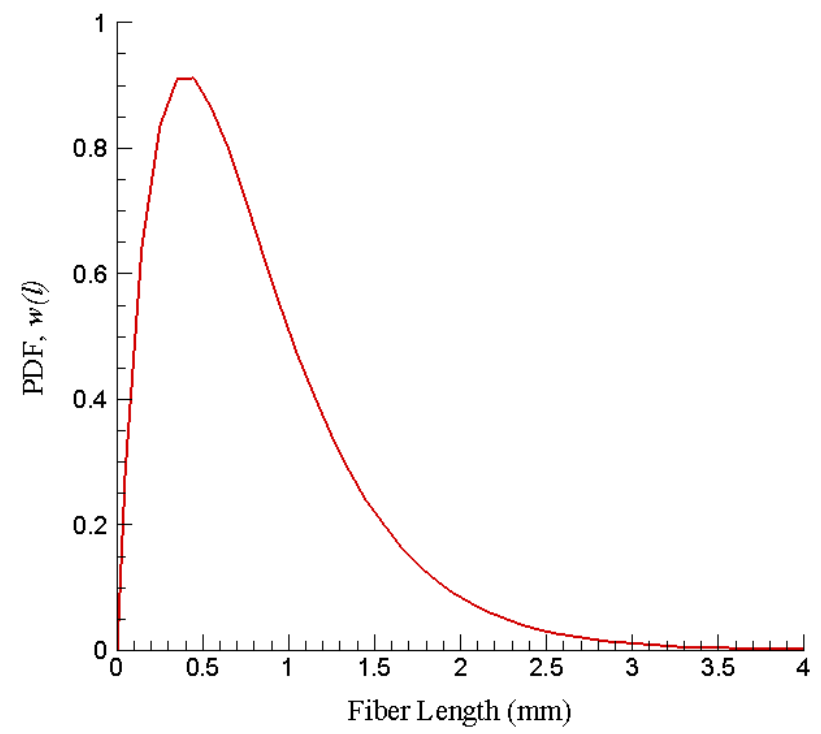

Figure 13. Assumed fiber length distributions for the EMTA calculation of the elastic properties for injection-molded carbon-fiber thermoplastics.

The EMTA calculations of the elastic properties for different injection-molded carbon-fiber thermoplastics based on the fiber orientation distribution presented in Figure 6 and the fiber length distribution given in Figure 13 are given in Tables 3 and 4 for 20\% and 30\% fiber volume fraction, respectively. The carbon/PEEK achieve the highest elastic moduli.

\begin{tabular}{|c|c|c|c|}
\hline $\begin{array}{c}\text { Property } \\
(\mathrm{MPa})\end{array}$ & Carbon/PP & Carbon/PA 6,6 & Carbon/PEEK \\
\hline$E_{11}$ & 12169 & 15998 & 18257 \\
\hline$E_{22}$ & 9824 & 13149 & 15233 \\
\hline$E_{33}$ & 3270 & 4876 & 6925 \\
\hline$G_{12}$ & 3627 & 4939 & 5695 \\
\hline$G_{13}$ & 960 & 1726 & 2257 \\
\hline$G_{23}$ & 909 & 1651 & 2170 \\
\hline
\end{tabular}

Table 3. EMTA elastic property results for the injection-molded carbon/PP, carbon/PA 6,6, and carbon/PEEK. 20\% fiber volume fraction was used in the computations. 


\begin{tabular}{|c|c|c|c|}
\hline $\begin{array}{c}\text { Property } \\
(\mathrm{MPa})\end{array}$ & Carbon/PP & Carbon/PA 6,6 & Carbon/PEEK \\
\hline$E_{11}$ & 18256 & 23235 & 25957 \\
\hline$E_{22}$ & 14569 & 18813 & 21274 \\
\hline$E_{33}$ & 3847 & 5566 & 7785 \\
\hline$G_{12}$ & 5407 & 7097 & 8011 \\
\hline$G_{13}$ & 1234 & 2131 & 2724 \\
\hline$G_{23}$ & 1152 & 2011 & 2586 \\
\hline
\end{tabular}

Table 4. EMTA elastic property results for the injection-molded carbon/PP, carbon/PA 6,6, and carbon/PEEK. 30\% fiber volume fraction was used in the computations.

\subsection{EMTA Computation of Elastic Properties for Compression-Molded Fiber Thermosets}

As injection-molded short-/long-fiber thermoplastics generally present low to moderate stiffness values, they cannot be used in important load-bearing structures. This stiffness limitation is mainly due to the limited fiber volume fraction that can be achieved for injectionmolded fiber materials. In addition, it is highly challenging to conserve fiber length during injection molding. Fiber length degradation also results in low mechanical properties. Although the LFT composite stiffness can reach the stiffness limit for fiber length, other mechanical properties such as strength, impact properties, fatigue and creep endurances do require significantly longer fiber length than the actual residual length. For important hydropower structural applications that require high stiffness, it is necessary to look for other types of discontinuous fiber polymer composites that can satisfy the stiffness requirement. Compressionmolded fiber thermosets could be a good solution as it is possible to achieve high fiber volume fraction (up to 60\%) and conserve fiber length during molding.

The EMTA computational procedure to calculate the elastic properties of a compressionmolded fiber polymer composite is depicted in Figure 14 that includes three steps. Step 1 involves the calculations of the elastic properties of the fiber tow. The tow contains unidirectional fibers with very high fiber volume fraction. The EMT homogenization procedure for high concentration is used to compute the tow elastic properties. The tows behave like equivalent fibers having the tow aspect ratio and homogenized properties. Next, the EMT model for high concentration is used to determine the elastic properties of the unidirectional composite (Step 2) that is composed of unidirectional fiber tows embedded in the matrix material. Finally, the elastic properties of the actual random fiber composite are computed from the properties of the unidirectional composite (calculated in Step 2) that are averaged over all possible orientations using the orientation averaging method [18] that makes use of orientation tensors.

Table 5 presents the EMTA results for the elastic properties of the carbon and glass fiber tows. The elastic modulus and Poisson ratio of the epoxy resin used are $4140 \mathrm{MPa}$ and 0.35 , respectively. The elastic properties of glass fiber and of carbon fibers are given in Tables 1 and 2. Both glass and carbon fiber tows are assumed to have a fiber volume fraction of $72 \%$ while the resulting compression-molded composite has a total fiber volume fraction that is varied to 
study the effect of the total fiber volume fraction on the composite elastic properties. To estimate the tow aspect ratio, we consider the $12 \mathrm{~K}$ (12,000 filaments) carbon fiber tow being 50.8-mmlong, this produces a tow aspect ratio of 57. For a comparison of the elastic properties for the resulting composites, the same glass fiber tow aspect ratio is used in the computation.

Two-dimensionally random fiber orientation is assumed in the compression-molded composite, this assumption leads to $A_{11}=A_{22}=0.5$, other components of the second-order orientation tensor are zero.
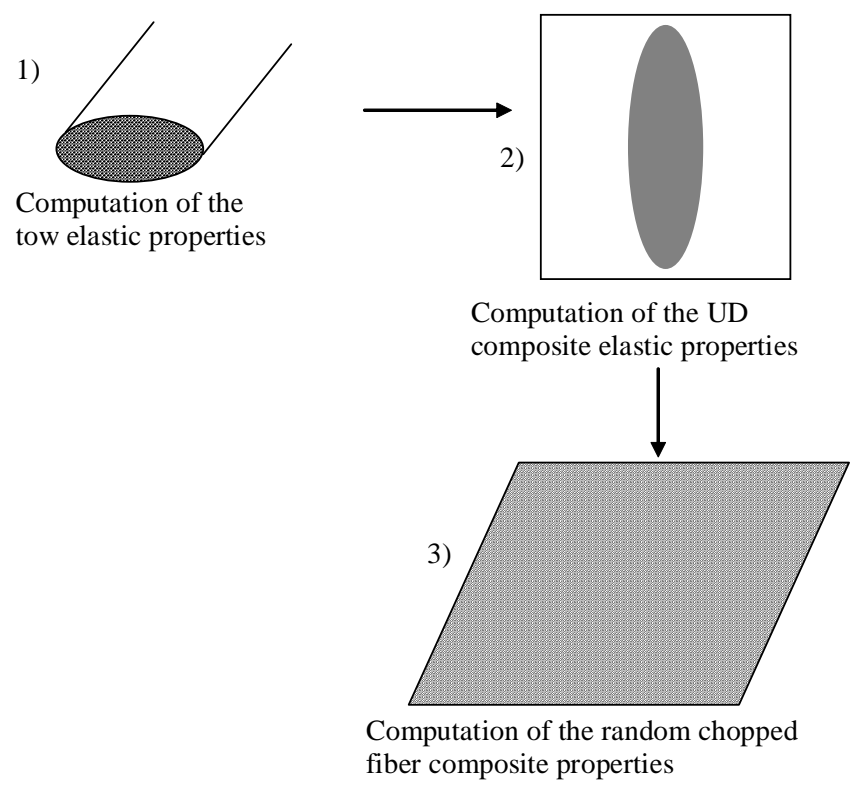

Figure 14. Schematic picture describing the computational procedure to predict the elastic properties of a compression-molded fiber polymer composite.

\begin{tabular}{|c|c|c|}
\hline & Carbon Fiber Tow & Glass Fiber Tow \\
\hline$E_{11}^{\text {tow }}(\mathrm{MPa})$ & 167560 & 53287 \\
\hline$E_{22}^{\text {tow }}=E_{33}^{\text {tow }}(\mathrm{MPa})$ & 11802 & 31082 \\
\hline$G_{12}^{\text {tow }}=G_{13}^{\text {tow }}(\mathrm{MPa})$ & 9071 & 16532 \\
\hline$G_{23}^{\text {tow }}(\mathrm{MPa})$ & 4490 & 15380 \\
\hline$v_{12}^{\text {tow }}=v_{13}^{\text {tow }}$ & 0.22 & 0.22 \\
\hline$v_{23}^{\text {tow }}$ & 0.29 & 0.3 \\
\hline
\end{tabular}

Table 5. Elastic properties for the carbon fiber tow and glass fiber tow predicted by EMTA. 
Figures 15 and 16 show the predicted elastic moduli as a function of the total fiber volume fraction for the compression-molded glass and carbon fiber composites. High modulus values are obtained using carbon fibers. However, comparative modulus values can also be obtained using glass fibers with higher fiber volume fractions. For instance, to achieve $30 \mathrm{GPa}$ for the elastic moduli $E_{11}$ and $E_{22}$, about $30 \%$ carbon fiber volume fraction is necessary while the same modulus value can be attained using $56 \%$ glass fiber volume fraction. As carbon fibers are much more expensive than glass fibers, glass fibers could be used for structural applications where the structure's weight is not a primordial factor for the design. Comparing Figure 15 to Figure 12 for injection-molded composites clearly shows that significantly higher elastic moduli can be achieved with compression-molded materials.

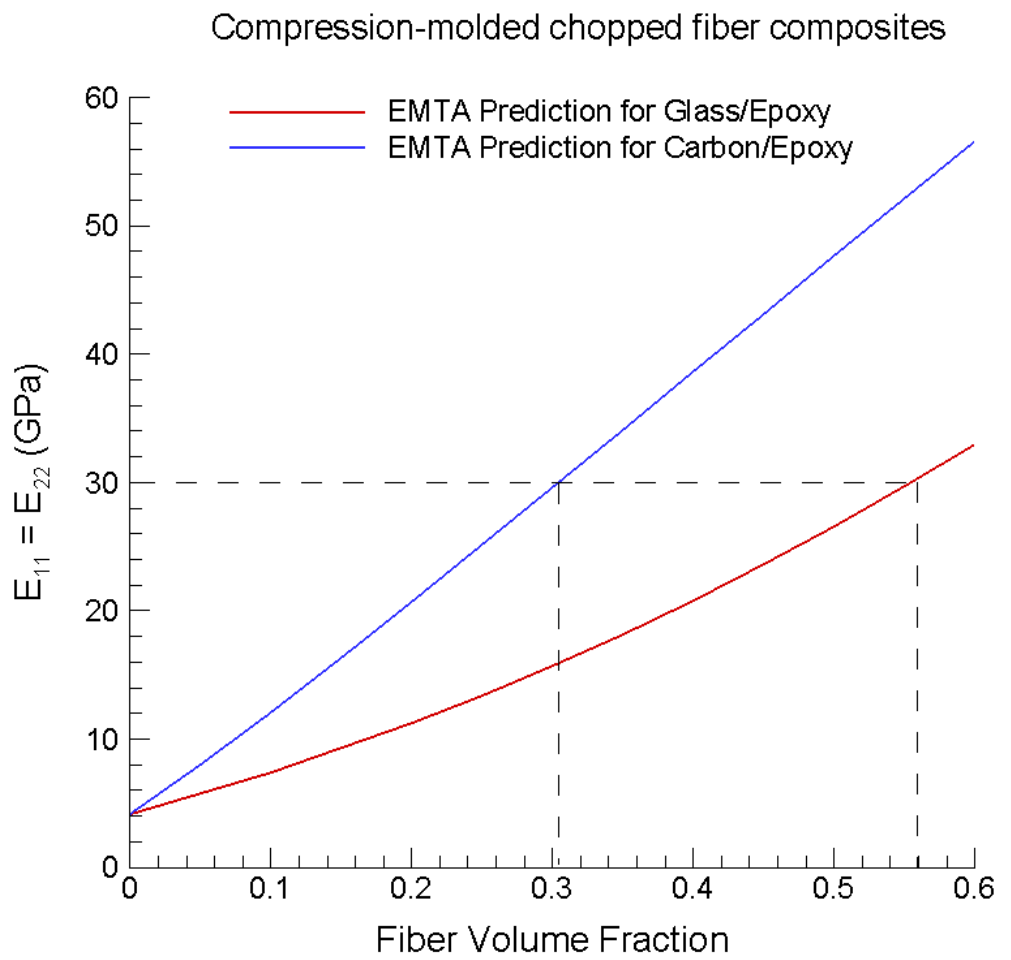

Figure 15. Predicted elastic moduli $E_{11}=E_{22}$ for compression-molded chopped fiber composites. 


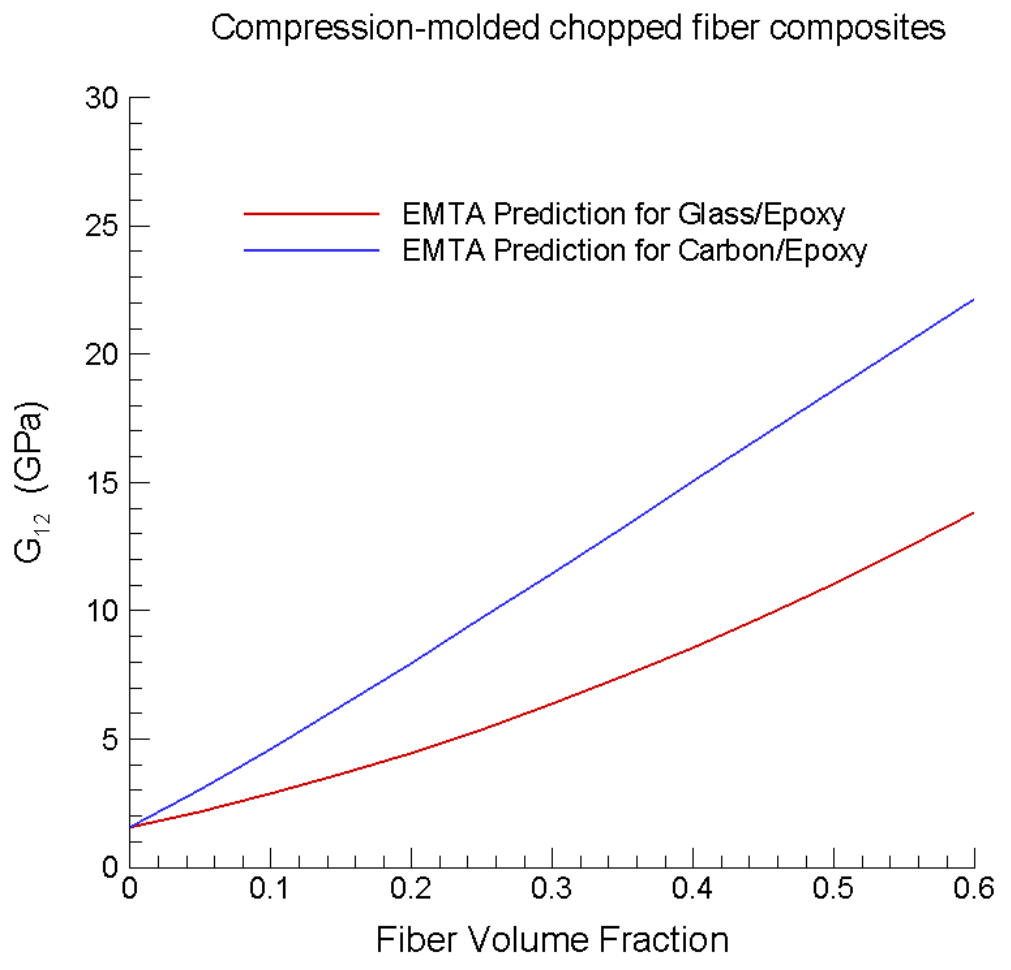

Figure 16. Predicted elastic modulus $G_{12}$ for compression-molded chopped fiber composites.

\section{Conclusions}

The EMTA code has been applied in this report to evaluate the elastic stiffness of discontinuous fiber polymer composites that can be processed by injection molding or compression molding in order to determine their potential uses in hydropower systems. Both glass and carbon fibers as well as both thermoplastic and thermoset resins have been examined. Injection molding of fiber thermoplastics can produce composite parts in a very fast and costeffective manner. However, because of the limit of the fiber volume fraction that can be achieved by injection molding, relatively low values of the composite stiffness are obtained. The use of carbon fibers has improved the composite properties very significantly. Also, the selection of the thermoplastic resin can improve the composite properties. The results show that the use of a PEEK matrix allows considerable gain in elastic properties compared to the other popular thermoplastics such as PP or PA6,6. However, the PEEK resins and fiber/PEEK pellets are more expensive, and therefore, it would be preferable to look for other types of resins and processing methods. Because of low stiffness values, injection-molded composites would be suitable for applications in the secondary structures that would not bear the loading from the water.

Compression-molded composites on the other hand offer significantly higher stiffness values due to high fiber volume fractions and conservation of fiber length that can be achieved by compression molding. Based on the range of the predicted stiffness values for compressionmolded materials, these materials would be considered for potential applications in load-carrying 
components of hydropower systems. As it is possible to increase the fiber volume fraction up to $60 \%$, the use of glass fiber could produce as good composite stiffness as the use of carbon fibers with lower fiber volume fractions. Therefore, glass fibers present a good alternative to carbon fibers in case lower material costs are sought and weight is not a decisive design factor.

\section{Acknowledgements}

This work has been funded by the US Department of Energy's Office of Wind and Hydropower Technologies. The development of the EMTA computer software was partly funded by the US Department of Energy's Office of Vehicle Technologies. The author would like to thank Mr. Kevin Simmons, Senior Research Scientist at PNNL, for providing Figures 1 and 2 illustrating PNNL’s injection molding and compression molding machines.

\section{References}

[1] Eshelby, J.D (1957). "The Determination of the Elastic Field of an Ellipsoidal Inclusion and Related Problems.” Proceedings of the Royal Society London, A 241: 376-396.

[2] Mori, T. and K. Tanaka (1973). "Average Stress in Matrix and Average Elastic Energy of Materials with Misfitting Inclusions.” Acta Metallurgica, 21:571-574.

[3] Benveniste, Y. (1987). "A New Approach to the Application of Mori-Tanaka's Theory in Composite Materials.” Mechanics of Materials, 6:147-157.

[4] Tucker III, C.L. and Liang, E. (1999). "Stiffness Predictions for Unidirectional Short-Fiber Composites: Review and Evaluation.” Composites Science and Technology, 59: 655-671.

[5] Nguyen, B.N., Tucker, B.J. and Khaleel, M.A. (2005). "A Mechanistic Approach to Matrix Cracking Coupled with Fiber-Matrix Debonding in Short-Fiber Composites.” Journal of Engineering Materials and Technology, 127: 337-350.

[6] Nguyen, B.N., Bapanapalli, S.K., Holbery, J.D., Smith, M.T., Kunc, V., Frame, B.J., Phelps, J.H. and Tucker III, C.L. (2008). "Fiber Length and Orientation Distributions in Long-Fiber Injection-Molded Thermoplastics - Part I: Modeling of Microstructure and Elastic Properties.” Journal of Composite Materials, 42:1003-1029.

[7] Bapanapalli, S.K. and Nguyen, B.N. (2008). "Prediction of Elastic Properties for Curved Fiber Polymer Composite.” Polymer Composites, 29(5):544-550.

[8] Nguyen, B.N., Simmons, K.L., Johnson, K.I., and Holbery, J.D. (2007). “The Effect of Fiber Orientation on the Elastic Properties of Discontinuous Fiber Composites.” In Proceedings of $22^{\text {nd }}$ Technical Conference of the American Society for Composites, P. Feraboli and P. Stickler, eds, DEStech Publications, Lancaster, PA.

[9] Selzer, R. and Friedrich, K. (1997). "Mechanical Properties and Failure Behaviour of Carbon Fibre-reinforced Polymer Composites under the Influence of Moisture.” Composites: Part A, 28A:595-604.

[10] Kootsookos, A. and Mouritz, A.P. (2004). "Seawater Durability of Glass- and CarbonPolymer Composites.” Composites Science and Technology, 64:1503-1511.

[11] Cartie, D., Davies, P., Peleau. M., and Partridge, I.K. (2006). "The Influence of Hydrostatic Pressure on the Interlaminar Fracture Toughness of Carbon/Epoxy Composites.” Composites: Part B, 37:292-300. 
[12] Guell, D.C. and Benard, A. (1997). "Flow-induced Alignment in Composite Materials: Current Applications and Future Prospects." In: Flow-induced Alignment in Composite Materials, T.D. Papathanasiou \& D.C. Guell, eds, pp 1-42, Woodhead Pulishing Limited, Cambridge, England.

[13] Murray, A.D. (1987). “Injection Molding.” In Composites, Vol. 1: Engineered Materials Handbook, pp. 555-558. ASM International, Metals Park, OH.

[14] Johnson, C.F. (1987). “Compression Molding." In Composites, Vol. 1: Engineered Materials Handbook, pp. 559-563. ASM International, Metals Park, OH.

[15] Schjødt-Thomsen, J. and R. Pyrz (2001). "The Mori-Tanaka Stiffness Tensor: Diagonal Symmetry, Complex Fiber Orientations and Non-Dilute Volume Fractions.” Mechanics of Materials, 33: 531-544.

[16] Mura, T. (1987). "Micromechanics of Defects in Solids," Second Edition, Martinus Nijhoff Publisher, Hingham, MA.

[17] Chen, C.-H. and C.-H. Cheng (1996). "Effective Elastic Moduli of Misoriented ShortFiber Composites.” International Journal of Solids and Structures, 33: 2519-2539.

[18] Advani, S. and Tucker III, C.L. (1987). "The Use of Tensors to Describe and Predict Fiber Orientation in Short-Fiber Composites.” Journal of Rheology, 31: 751-784.

[19] Thomason, J.L. (2002). "Interfacial Strength in Thermoplastic Composites - at last an Industry Friendly Measurement Method?” Composites: Part A, 33:1283-1288.

[20] Rae, P.J., Brown, E.N., and Orler, E.B. (2007). "The Mechanical Properties of Poly(ether-ether-ketone) (PEEK) with Emphasis on the Large Compressive Strain Response.” Polymer, 48:598-615. 